\title{
GATA4 regulates osteogenic differentiation by targeting miR-144-3p
}

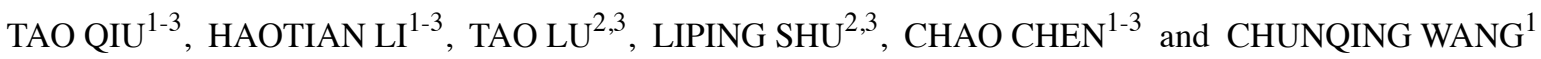 \\ ${ }^{1}$ Department of Orthopedic Trauma, The Affiliated Hospital of Guizhou Medical University; \\ ${ }^{2}$ Center for Tissue Engineering and Stem Cell Research, ${ }^{3}$ National-Local Joint Engineering Laboratory of Cell Engineering \\ and Biomedicine, Guizhou Medical University, Guiyang, Guizhou 550004, P.R. China
}

Received November 4, 2020; Accepted June 3, 2021

DOI: $10.3892 /$ etm.2021.11006

\begin{abstract}
Numerous studies have demonstrated that microRNAs (miRNAs or miRs) play an important role in regulating osteogenic differentiation, but their specific regulatory mechanism requires further investigation. In the present study, it was revealed that during osteogenic differentiation of rat bone marrow mesenchymal stem cells (BMSCs), the expression level of miR-144-3p was decreased with increased osteogenic induction duration and was negatively associated with osteogenic marker gene expression. Overexpression of miR-144-3p inhibited osteogenic differentiation, while inhibition of miR-144-3p expression promoted osteogenic differentiation. In addition, dual-luciferase activity analysis and adenovirus infection experiments revealed that GATA binding protein 4 targeted miR-144-3p for regulation and that overexpression of GATA4 promoted the expression of miR-144-3p. These data indicated that miR-144-3p plays a role in inhibiting BMSC osteogenic differentiation and that GATA4 inhibits osteogenic differentiation by targeting miR-144-3p expression.
\end{abstract}

\section{Introduction}

Osteoporosis (OP) is characterized by reduced bone formation, increased bone resorption, and destruction of the bone microstructure, resulting in reduced bone strength, increased brittleness and metabolic osteopathic syndrome, which predisposes patients to fractures $(1,2)$. Currently, the effects of OP treatments are not ideal, and additionally, more detailed basic research studies are necessary to identify a new target for treatment.

Bone marrow mesenchymal stem cells (BMSCs) are a subgroup of adult stem cells with multiple differentiation

Correspondence to: Professor Chunqing Wang, Department of Orthopedic Trauma, The Affiliated Hospital of Guizhou Medical University, 28 Guiyi Street, Guiyang, Guizhou 550004, P.R. China E-mail: wangchunqing@gmc.edu.cn

Key words: bone marrow mesenchymal stem cells, osteogenic differentiation, GATA binding protein 4, microRNA-144-3p potential found in the bone marrow matrix of mammals. BMSCs exhibit the potential to differentiate into a variety of cell types, high plasticity, the easy introduction and expression of exogenous genes and other advantageous biological characteristics and can differentiate into osteoblasts in an osteoblast-induced microenvironment $(3,4)$. Due to this characteristic, BMSCs play an important role in the basic research of OP.

MicroRNAs (miRNAs or miRs), a type of endogenous single-stranded noncoding small RNA that has been revealed in previous studies to be widespread in animals and plants, are important posttranscriptional regulators $(5,6)$. In previous studies, miRNAs have been revealed to be involved in the regulation of a variety of physiological and pathological processes in the body and have gradually become the focus of life science research $(7,8)$. miRNAs play an important regulatory role in the proliferation and differentiation of osteoblasts, osteoclasts and chondrocytes by regulating the expression of target genes through relevant signaling pathways, ultimately affecting bone production and metabolism $(9,10)$. miRNAs are expected to become potential gene therapy targets for the clinical treatment of bone metabolic diseases and bone injury. Previous studies have found stable miRNAs in circulating blood and identified significantly different expression levels of various miRNAs in the peripheral blood of patients with OP compared with those in healthy subjects $(11,12)$. Our previous study demonstrated that the expression of miR-144-3p in the peripheral blood and bone tissues of OP patients was significantly decreased (13).

Members of the GATA family of transcription factors are highly evolutionarily conserved, zinc finger domain-specific transcription factors that bind a specific DNA sequence, $(\mathrm{A} / \mathrm{T})$ $\operatorname{GATA}(\mathrm{A} / \mathrm{G})$, and regulate a variety of biological processes. GATA binding protein 4 (GATA 4) is one of six transcription factors in the family (14). Most previous studies of GATA4 have focused on its role in the heart $(15,16)$. Another previous study also revealed that GATA4 plays an important role in the regulation of bone metabolism, especially in the differentiation and formation of osteoblasts (17). After consulting the literature and miRNA Base (13), it was hypothesized that GATA4 is an upstream targeted regulator of miR-144-3p.

In the present study, the role of miR-144-3p in regulating the osteogenic differentiation of BMSCs and the effect of 
GATA4 on osteogenic differentiation by targeting miR-144-3p was demonstrated.

\section{Materials and methods}

Animals. A total of six male Sprague-Dawley rats aged 2-3 weeks and weighing 40-60 g, were obtained from the Experimental Animal Centre of Guizhou Medical University, (Guizhou, China) and were used in the present study. The rats were kept in a temperature-controlled room, with a humidity of $40-70 \%$, in a $12 \mathrm{~h}$ light-dark cycle with free access to standard chow and tap water. All animals were reared in a specific pathogen-free environment at a comfortable temperature and humidity (18). The BMSC extraction process was approved by the Experimental Animal Ethics Committee of Guizhou Medical University (approval no. 1702032).

Identification rat BMSCs by using flow cytometry. To identify the target cell, fluorescein isothiocyanate (FITC)-conjugated CD90 (1:100; cat. no. 561973; BD Biociences), CD45 (1:100; cat. no. 561867; BD Biosciences), and phycoerythrin (PE)-conjugated CD 44 (1:100; cat. no. MA5-16908; Thermo Fisher Scientific Inc.) were used to label the BMSC membranes. A flow cytometer (FC 500; BD Biosciences) and FlowJo software v.10.5.2 (BD Biosciences) were used for analysis.

Mesenchymal stem cell culture and osteogenic differentiation. The isolation and culture of BMSCs were conducted as previously described (13). Briefly, the rats were weighed and $150 \mathrm{mg} / \mathrm{kg}$ of pentobarbital sodium were administered intraperitoneally for euthanasia. After euthanasia, BMSCs were isolated from bilateral femurs and tibia of rats and cultured in a cell incubator containing $5 \% \mathrm{CO}_{2}$ at $37^{\circ} \mathrm{C}$. Medium consisting of Dulbecco's modified Eagle's medium (DMEM; Gibco; Thermo Fisher Scientific, Inc.), $10 \mathrm{ml}$ of fetal bovine serum (FBS; Biological Industries), and $1 \mathrm{ml}$ of secondary antibody (cat. no. 30-002-CI; Beijing Solarbio Science \& Technology Co., Ltd.), at a ratio of 100:10:1 was prepared and replaced every 2-3 days. When the cells reached $80 \%$ confluence, they were digested with a trypsin-EDTA solution (Beijing Solarbio Science \& Technology Co., Ltd.) and passaged at a ratio of 1:2.

Osteogenic induction medium was prepared by adding $10 \% \mathrm{FBS}, 100 \mathrm{mmol} / \mathrm{l}$ dexamethasone, $0.05 \mathrm{mmol} / \mathrm{l}$ vitamin C, and $10 \mathrm{mmol} / \mathrm{l}$ glycerophosphate (all purchased from Beijing Solarbio Science \& Technology Co., Ltd.) to $90 \mathrm{ml}$ of high-glucose DMEM and stored at $4{ }^{\circ} \mathrm{C}$. During in vitro osteogenic differentiation, cells were seeded in 6-well plates and induced by osteogenic induction medium, which was replaced every 2-3 days.

Alkaline phosphatase (ALP) and Alizarin Red S staining. BMSCs from generations 4 and 5 were inoculated in 6-well plates, and osteogenic induction medium was added when the cells reached 70-80\% confluence for induction. Alizarin Red S (Beijing Solarbio Science \& Technology Co.,Ltd.) staining was then carried out on days $0,7,14$ and 21 strictly according to the manufacturer's protocol. The medium in the plate was first discarded, and the cells were washed 3 times with PBS. After fixation with $4 \%$ paraformaldehyde for $10-15 \mathrm{~min}$ at room temperature, the fixation solution was discarded and the cells were washed 3 times with $\mathrm{ddH}_{2} \mathrm{O}$. After dd (double distilled) $\mathrm{H}_{2} \mathrm{O}$ was completely absorbed, an $4 \%$ Alizarin Red S staining solution was slowly added and incubated with the cells at $37^{\circ} \mathrm{C}$ in a humidified atmosphere of $5 \% \mathrm{CO}_{2}$ for $20-30 \mathrm{~min}$. The dye was discarded and the cells were washed 3-5 times with $\mathrm{ddH}_{2} \mathrm{O}$. The appropriate amount of $\mathrm{ddH}_{2} \mathrm{O}$ was added to each well to prevent the cells in the well from drying. The cells were observed, and images were captured under a light microscope (Leica DMi8 M/C/A; Leica Microsystems, GmbH) (magnification, x100).

An ALP staining kit (Beyotime Institute of Biotechnology) was used for staining according to the manufacturer's instructions. The medium in 6-well plates was discarded, and cells were washed 3 times with PBS. The reagent (75\% fixative solution) was used to fix the cells for 2-5 min at room temperature, and the cells dried naturally. The fixative solution was discarded, and the cells were rinsed with distilled water for $30 \mathrm{sec}$. After the distilled water had been discarded, the matrix solution was added, and the cells were incubated at $37^{\circ} \mathrm{C}$ in the dark for $15 \mathrm{~min}$. After the excess dye had been absorbed, $200 \mu \mathrm{l}$ color solution A was immediately added, followed by incubation for $5 \mathrm{~min}$ at room temperature and washing for $30 \mathrm{sec}$. The excess water was discarded, and $10 \mu \mathrm{l}$ color solution B was added, followed by incubation for $5 \mathrm{~min}$ at room temperature and washing for $30 \mathrm{sec}$. After the excess water had been discarded, dye was added for $30 \mathrm{sec}$, followed by a washing step for $30 \mathrm{sec}$, after which the excess water was discarded. The cells were then observed under a light microscope (Nikon TE-2000; Nikon Corporation) (magnification, $\mathrm{x} 40$ ) and images were captured.

Transfection assays. All transfection reagents [the miR-144-3p oligonucleotides (miR-144-3p mimic 5'-UACAGUAUAGAU GAUGUACU-3', negative mimic control 5'-UUCUCCGAA CGUGUCACGUTT-3, miR-144-3p inhibitor 5'-AGUACA UCAUCUAUACUGUA3', inhibitor negative control 5'-CAG UACUUUUGUGUAGUACAA) and GP-siRNA-Mate Plus] were purchased from Shanghai GenePharma Co., Ltd. BMSCs at generations 4-5 were digested, seeded into 6-well plates at a density of $3 \times 10^{5}$ cells $/ \mathrm{ml}$ and cultured to a confluence of $60-80 \%$ for transfection. Prior to transfection, the transfection reagent (RNA oligo) was prepared at the desired concentration $(4 \mu \mathrm{l})$ according to the manufacturer's instructions, mixed directly with the GP-siRNA-Mate Plus transfection reagent at room temperature, and left to rest for $10-15 \mathrm{~min}$. The compound was added to $300 \mu \mathrm{l}$ of complete medium, mixed and added to a 6-well plate. After 6-8 h, this solution was added to complete medium or osteogenic induction medium. mRNA and protein expression was detected at 36-72 $\mathrm{h}$ after transfection. For each group of tests, 3 wells containing the compound were prepared, and the mean value was obtained. The experiment was repeated three times.

Adenovirus infection. The cells were inoculated into a 48-well plate at a density of $1 \times 10^{5}$ cells/well. A total of $250 \mu 1$ of DMEM containing 10\% FBS, $100 \mu \mathrm{l}$ adenovirus (Shanghai GenePharma Co., Ltd) and $100 \mu \mathrm{l}$ Lipofectamine $2000^{\circledR}$ reagent (Invitrogen; Thermo Fisher Scientific, Inc) was added to each well. After mixing, the cells were cultured for $24 \mathrm{~h}$ 
Table I. Gene sequences of plasmid P1.

pGL3-miR-144-p1 (568 bp)

CTAGCATCTTCAGCCTTCAGTTTCATTCCCAGCACAGGAAACTAAGCAGA

AGATAAACAAAAAGGGAGCCAAGCCTCAGCTTGTCTCAGGAAGCCAGC

AGGCAAAGAGTTAAGAAGCAGGGACTTCTAGAACCCGGGAAAACGTGC

CCCACCCAGGGGAGGGGCCAGAGGGTTAAAAGCCAAGCTGCTTGAGTG

AGAAGAGACAAGGCAGGCTCTCCCTGTGCAGAGGATTCCCTGGACGAG

GCTCCAGCTCCACTCCAGCTCCAGGTAAGCAGTCCTTGGAGTGGCTGTC

AGCCTGCTTATAGGTCTGCCCAGAGGGAAGCTCCTGCCTCACAACTTCGT

TTCTGCCTGTAACTCTGGATCCCTAAGAGACCCGAGTAGACCTTAGCTTC

CTTCTCTAAGCCACCTGGGGTTATCCTGGACCACAGGATCAGGGAGATGC

TGCTCTGGGAGGGAAGTGGAGGAGCAGAGGTAGGGACTTAGGTGTCCC

TGACTGACCCTGAGCCAATCCCCTGGCTCACTCCAGGCCTGCTGCTCAC

CTCCTCCTCCAGGACCTTGGCTGGGATATC

miR, microRNA.

in an incubator containing $5 \% \mathrm{CO}_{2}$ at $37^{\circ} \mathrm{C}$. The adenovirus with AdGATA4 (Shanghai GenePharma Co., Ltd.) in the 48-well plate was absorbed and $500 \mu \mathrm{l}$ of DMEM containing $10 \%$ FBS was added to each well, followed by continued culture at $37^{\circ} \mathrm{C}$ in an incubator containing $5 \% \mathrm{CO}_{2}$ for $48 \mathrm{~h}$. After 48 h, RT-qPCR for detection of GATA binding protein 4 and miR-144-3p expression was performed. The adenovirus infection efficiency was observed under a confocal microscope (Nikon eclipse 80i; Nikon Corporation) (magnification, x40). $\mathrm{B}$ was the blank control group (untransfected cells).

Dual-luciferase reporter assay. For luciferase reporter assays, three plasmids were designed: Plasmids containing one cis-GATA4 motif from the miR-144 promoter (P1) or two cis-GATA4 motifs from the miR-144 promoter (P2) and the pGL3-basic vector. The plasmids were purchased from Shanghai GenePharma Co., Ltd. and the sequences are listed in Tables I and II. After 293T cells (Shanghai Furi Technology Co., Ltd.) had been cultured in a $10-\mathrm{cm}$ culture dish until reaching $80-90 \%$ confluence, the cells were diluted to $1 \times 10^{6}$ cells $/ \mathrm{ml}$. The cells were used to inoculate 12 -well plates at a density of $5 \times 10^{5}$ cells/well, mixed and cultured for $24 \mathrm{~h}$ at $37^{\circ} \mathrm{C}$ in an atmosphere containing $5 \% \mathrm{CO}_{2}$. The culture medium in the 12-well plate was aspirated and the transfection mixture GP-transfect-Mate (Shanghai GenePharma Co., Ltd.) was added dropwise into the 12 -well plate at room temperature for $20 \mathrm{~min}$. After mixing, the mixture was incubated for $5 \mathrm{~h}$ at $37^{\circ} \mathrm{C}$ under $5 \% \mathrm{CO}_{2}$ for 24 and $48 \mathrm{~h}$, and samples were collected. The cells were used for dual-luciferase assays in which luciferase activity was assessed using the Dual-Glo Luciferase Assay System (Promega Corporation). Three replicates were performed for each group, the mean values were obtained, and the detection time-point was $48 \mathrm{~h}$. The experiment was repeated three times.

Reverse transcription-quantitative $(R T-q) P C R$ for $m R N A$ and miRNA. Cell samples to be collected were obtained, and the culture medium was discarded. Then, cells were washed with PBS, and TRIzol reagent (Thermo Fisher Scientific, Inc.) was used to extract total RNA from the cells. A total of $2 \mu \mathrm{g}$ of RNA was used for reverse transcription. The total RNA was converted to cDNA with a Custom Gene RT-qPCR Quantitation Kit according to the manufacturer's instructions (Shanghai GenePharma Co., Ltd). Quantitative PCR was performed using a Bio-Rad CFX 96 Touch real-time PCR system (Bio-Rad Laboratories, Inc.) and was executed using the TB Green Premix Ex Taq II (Shanghai GenePharma Co., Ltd.) with $2 \mu \mathrm{l}$ of cDNA template in a $25-\mu 1$ final reaction mixture $\left(95^{\circ} \mathrm{C}\right.$ for $30 \mathrm{sec} ; 95^{\circ} \mathrm{C}$ for $5 \mathrm{sec}$, $60^{\circ} \mathrm{C}$ for $30 \mathrm{sec}, 40$ cycles). The average threshold cycle $(\mathrm{Ct})$ for each gene was determined from triplicate reactions; the relative expression level of mRNA or miRNAs was normalized to that of the internal controls, $\beta$-actin or U6 using the $2^{-\Delta \Delta \mathrm{Cq}}$ method (19).

The primers specific for mRNAs were purchased from Shanghai GenePharma Co., Ltd. and the sequences are listed in Table III.

Western blot analysis. After $72 \mathrm{~h}$ of transfection, the cells were washed with PBS and lysed with RIPA lysis buffer (cat. no. KGP702; Nanjing KeyGen Biotech Co., Ltd.). Protein determination was performed using the bicinchoninic acid (BCA) method. Samples ( $20 \mu \mathrm{g} /$ well) were separated by 8 and 15\% SDS-PAGE. After electrophoresis, a PVDF membrane was immersed in methanol for $10 \mathrm{sec}$, and the gel and PVDF membrane soaked in methanol were immersed in rapid electrophoretic buffer for $10 \mathrm{~min}$. Non-specific binding was blocked with $5 \%$ non-fat skimmed milk in Tris-buffered saline plus $0.1 \%$ TBST (cat. no. T1081; Beijing Solarbio Science $\&$ Technology Co., Ltd.) at $25^{\circ} \mathrm{C}$ for $2 \mathrm{~h}$. Membranes were probed with primary antibodies overnight at $4^{\circ} \mathrm{C}$, including Runt-related transcription factor 2 (Runx2) polyclonal antibody (1:1,000; cat. no. ab23981; Abcam) and ALP monoclonal antibody (1:2,000; cat. no. ab194297; Abcam). $\beta$-actin (1:10,000; cat. no. A5441; Sigma-Aldrich; Merck KGaA) was used as a loading control. After washing with TBS containing 
Table II. Gene sequences of plasmid P2.

pGL3-miR-144-p2 (969 bp)

TCAGCTTCCCAGCAGAGGCCCACTTGTCCACGGACCTTGCCAGAGGTGG CTTGCAAGCTTCAGCTCTGCCCACCCAGCTCAAACAGAGTCAAAGCCTA GGGATGGAGTCAGGCTGAGGGTACATGGAGCCTGCTCCCAGATAGATTC CATCTAGGTCCAGTTGCCAGGACCTCCCTGTCCTATTCAGATTCAACTAA CATTCCCATCATCACCCACAACAAACTGGGACCTTTCAGCGAGGCCCGA ACAACTAAGCCCTGAAGGACGTGGTGAGGGTCTTTCTCTTTCCTAGCCC AGTGGGGTAGGCAGGTAACCTTCTTTGTGGGGTTGGGGGTGAATGGTCA CCCACTTAGAAGACGGGAGGCAGGGTGGCGGTGTAGTGTCAGTGTTGG GGTTCTTGGCTAGCATCTTCAGCCTTCAGTTTCATTCCCAGCACAGGAAA CTAAGCAGAAGATAAACAAAAAGGGAGCCAAGCCTCAGCTTGTCTCAG GAAGCCAGCAGGCAAAGAGTTAAGAAGCAGGGACTTCTAGAACCCGGG AAAACGTGCCCCACCCAGGGGAGGGGCCAGAGGGTTAAAAGCCAAGCT GCTTGAGTGAGAAGAGACAAGGCAGGCTCTCCCTGTGCAGAGGATTCCC TGGACGAGGCTCCAGCTCCACTCCAGCTCCAGGTAAGCAGTCCTTGGAG TGGCTGTCAGCCTGCTTATAGGTCTGCCCAGAGGGAAGCTCCTGCCTCAC AACTTCGTTTCTGCCTGTAACTCTGGATCCCTAAGAGACCCGAGTAGACC TTAGCTTCCTTCTCTAAGCCACCTGGGGTTATCCTGGACCACAGGATCAG GGAGATGCTGCTCTGGGAGGGAAGTGGAGGAGCAGAGGTAGGGACTTA GGTGTCCCTGACTGACCCTGAGCCAATCCCCTGGCTCACTCCAGGCCTG CTGCTCACCTCCTCCTCCAGGACCTTGGCTGGGATATC

miR, microRNA.

Table III. Primers used in reverse transcription-quantitative PCR.

\begin{tabular}{lll}
\hline Gene & \multicolumn{1}{c}{ Forward primer } & \multicolumn{1}{c}{ Reverse primer } \\
\hline Rat-miR-144-3p & GCAGAGTACAGTATAGATGATG & GTGCAGGGTCCGAGGT \\
rat-GATA4 & GGGCTGTCATCTCACTATGGG & TGGATAGCCTTGTGGGGAGA \\
rat-U6 & CAGCACATATACTAAAATTGGAACG & GTGCAGGGTCCGAGGT \\
rat-ALP & CGATGGCTTTGGTACGGAGT & TGCGGGACATAAGCGAGTTT \\
rat-RUNX2 & TGAGATTTGTAGGCCGGAGC & CTGAGGCGGTCAGAGAACAA \\
rat-ACTB & CGTAAAGACCTCTATGCCAACA & GGAGGAGCAATGATCTTGATCT
\end{tabular}

miR, microRNA; ALP, alkaline phosphatase; RUNX2, Runt-related transcription factor 2; ACTB, $\beta$-actin.

$0.1 \%$ Tween- 20 , the immobilized primary antibodies were incubated with a horseradish peroxidase-conjugated secondary goat anti-rabbit IgG antibody (1:2,000; cat. no. ab205718; Abcam) for $1 \mathrm{~h}$ at $25^{\circ} \mathrm{C}$. Then, SuperSignal West Pico chemiluminescent substrate (P0018S; Beyotime Institute of Biotechnology) and a full-function FR-1800 luminescence and fluorescence biological image analysis system (Shanghai Furi Technology Co., Ltd.) were used for chemiluminescence detection. Gel-Pro Analyzer software version 4.0 (Media Cybernetics, Inc.) was used for analysis and processing.

Statistical analysis. All data are expressed as the mean \pm standard deviation (SD). Statistical analyses were performed with SPSS (version 17.0; SPSS, Inc.). Comparisons between only two groups were performed by the Student's t-test. One-way or two-way ANOVAs, with Bonferroni's post hoc tests were performed for comparisons among multiple groups. $\mathrm{P}<0.05$ was considered to indicate a statistically significant difference.

\section{Results}

Expression of miR-144-3p is downregulated during osteogenic differentiation. Our previous study revealed that the expression of miR-144-3p in the serum and bone tissue of OP patients was significantly increased compared with that of healthy individuals (13). BMSCs in rats were induced to undergo osteogenic differentiation to investigate whether the expression of miR-144-3p would change during the osteogenic differentiation of BMSCs in rats. The result of flow cytometry demonstrated that BMSCs were positive for CD44 (95.48\%), 
Day 0
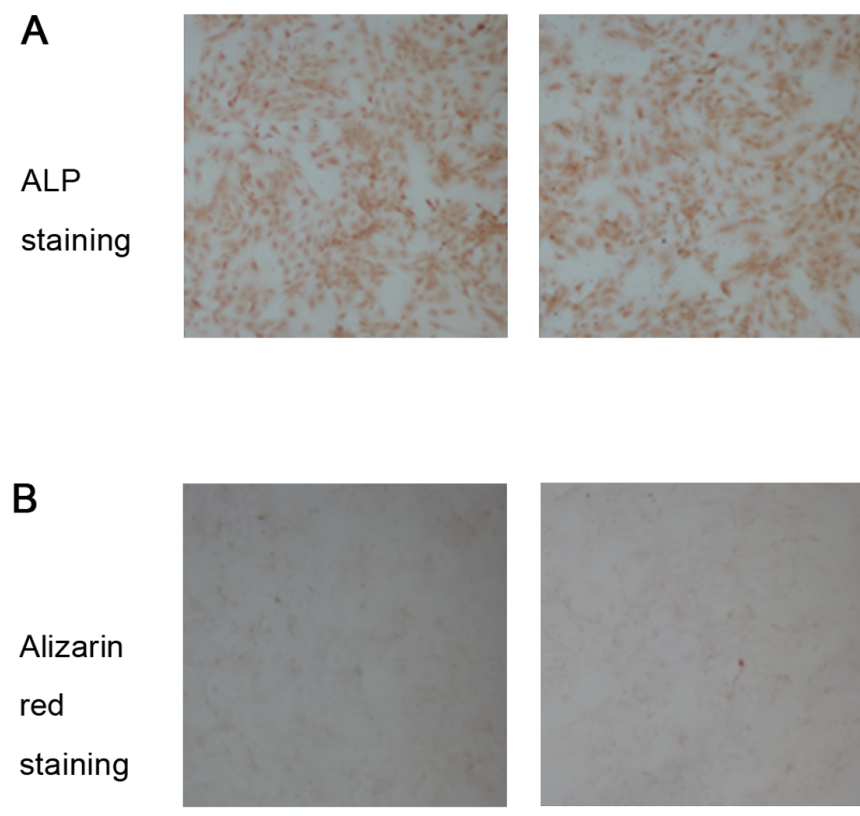
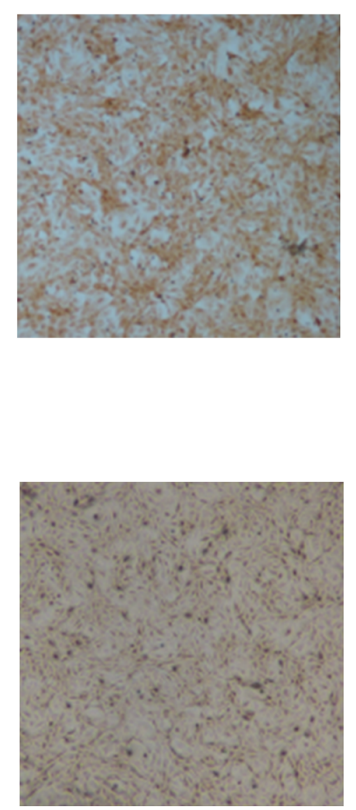

Day 21

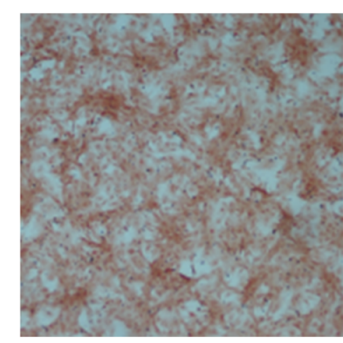

C

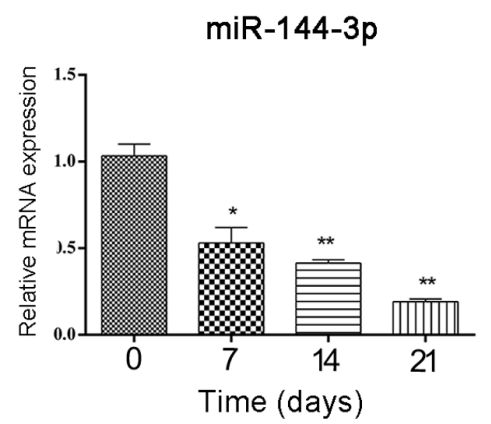

Day 14

Day 7

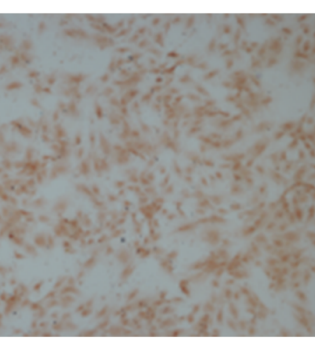

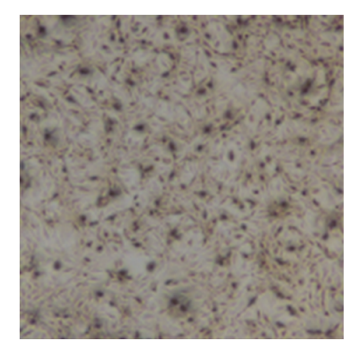

RUNX2
ALP

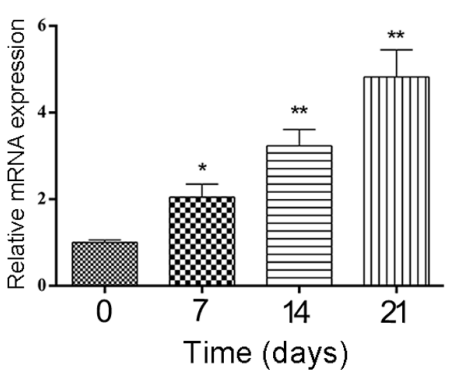

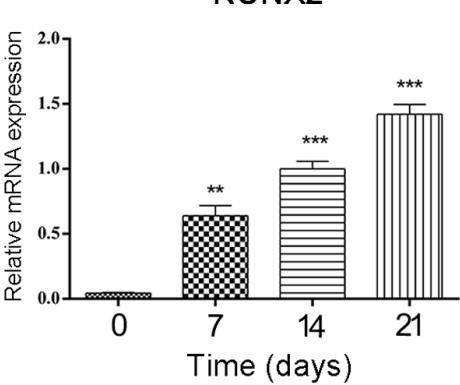

Figure 1. Expression of miR-144-3p is downregulated during osteogenic differentiation. (A and B) During osteogenic differentiation of BMSCs, ALP staining and Alizarin Red staining gradually deepened with time prolonging. The results were performed to ensure efficient osteogenic differentiation of BMSCs. (C) miR-144-3p, ALP, and Runx2 expression levels in BMSCs were quantitatively assessed by reverse transcription-quantitative PCR at the indicated time-points $(0,7,14$ and 21 days) during osteogenic differentiation. The data, normalized to $\beta$-actin (for mRNA) or U6 (for miRNA), are the mean \pm SD of three experiments. miR, microRNA. ${ }^{*} \mathrm{P}<0.05,{ }^{* *} \mathrm{P}<0.01$ and ${ }^{* * * *} \mathrm{P}<0.001$. ALP, alkaline phosphatase; BMSCs, bone marrow mesenchymal stem cells; RUNX2, Runt-related transcription factor 2.

CD29 (99.31\%) and negative for CD45 (0.20\%) (Fig. S1). During osteogenic differentiation of BMSCs, ALP staining and Alizarin Red staining gradually deepened with time prolonging (Fig. 1A and B). As induction duration increased, the mRNA expression of miR-144-3p was decreased, and the mRNA expression of the osteogenic marker genes ALP and Runx 2 was significantly increased (Fig. 1C). These results indicated that miR-144-3p was negatively associated with the expression of osteogenic marker genes.

miR-144-3p inhibits osteogenic differentiation. As previously demonstrated, miR-144-3p was involved in the osteogenic differentiation of BMSCs, but the specific regulatory role was not further examined. In this part of the study, the expression of miR-144-3p was regulated by cell transfection, and the relationship between the expression of miR-144-3p and osteogenic marker genes was analyzed to determine whether miR-144-3p negatively regulates the differentiation of osteoblasts.
The mRNA expression of miR-144-3p was assessed at $48 \mathrm{~h}$ after transfection in BMSCs transfected with miR-144-3p mimic, inhibitor and the corresponding controls. The expression of miR-144-3p mRNA in the transfected miR-144-3p mimic group was significantly increased compared with that in the corresponding negative control (NC) treatment group, while the expression of miR-144-3p mRNA in the miR-144-3p inhibitor group was significantly decreased compared with that in the inhibitor NC group (Fig. 2A). After BMSCs were transfected with the miR-144-3p mimic, the mRNA and protein expression of the osteogenic marker genes ALP and Runx2 were significantly decreased compared with that in the corresponding NC treatment group, while the mRNA and protein expression of the osteogenic marker genes ALP and Runx2 were significantly increased in the transfected miR-144-3p inhibitor group compared with the inhibitor NC group (Fig. 2A-C). These results indicated that miR-144-3p was a negative regulatory factor in the osteogenic differentiation 
A

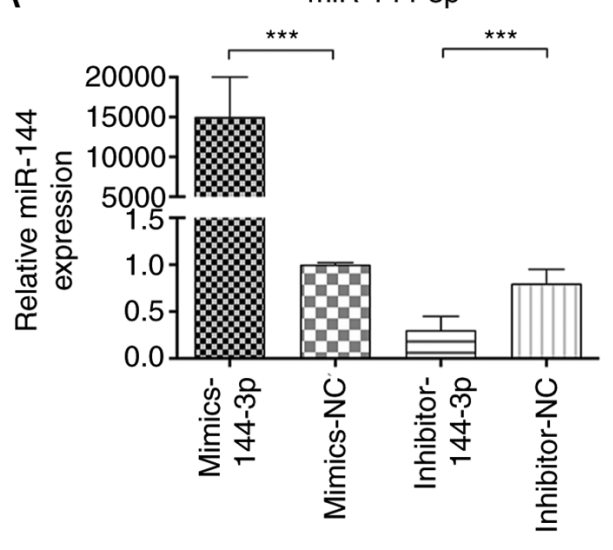

ALP

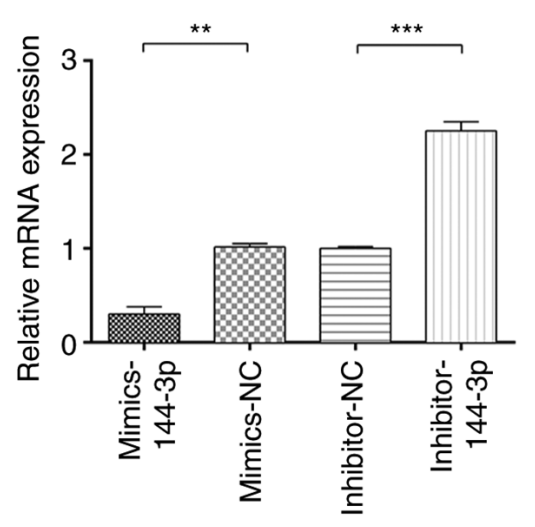

RUNX2

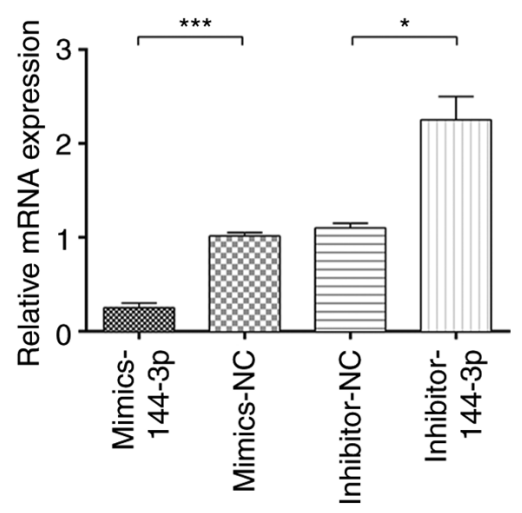

B

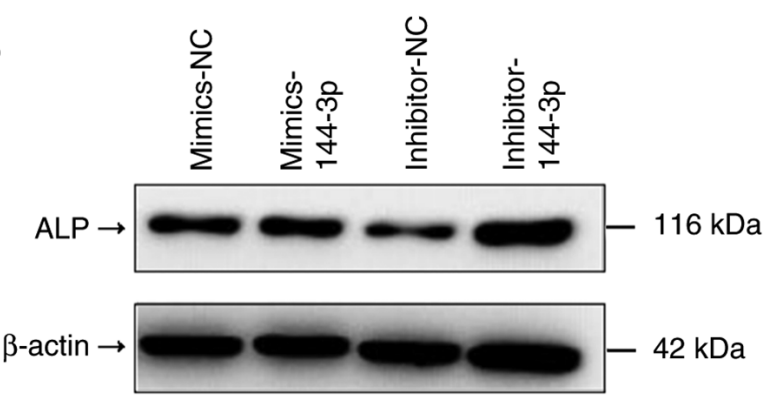

C

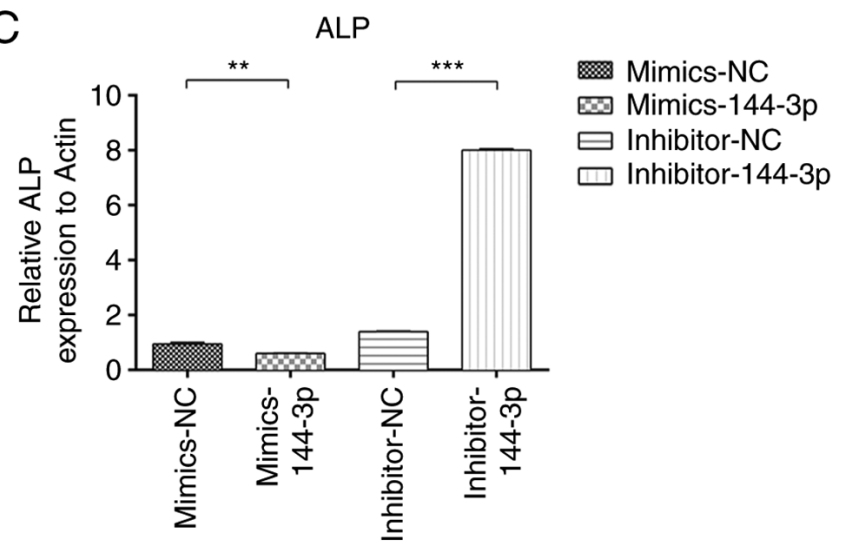

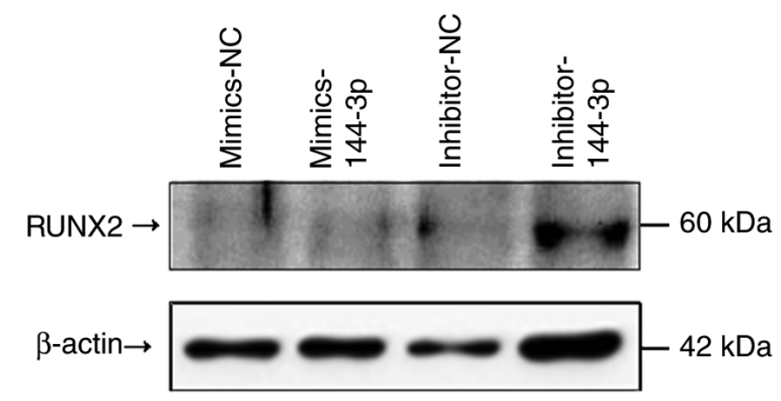

RUNX2

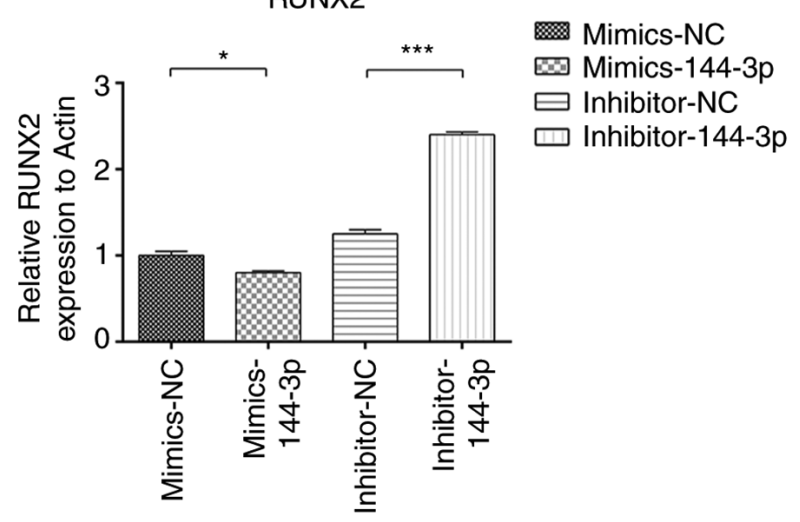

Figure 2. miR-144-3p inhibits osteogenic differentiation. (A) miR-144-3p mimic/miR-144-3p inhibitor or their corresponding negative controls were transfected into bone marrow mesenchymal stem cells. The mRNA levels of osteogenic markers (ALP and Runx2) and miR-144-3p were quantified by reverse transcription-quantitative PCR. $\beta$-actin (for mRNA) or U6 (for miRNA) were used as the internal controls. The values of each group are expressed as the mean $\pm \mathrm{SD}$ of three independent experiments performed in triplicate. (B and C) Protein levels of ALP and Runx 2 were analyzed by western blotting. ${ }^{*} \mathrm{P}<0.05$, ${ }^{* *} \mathrm{P}<0.01$ and $^{* * *} \mathrm{P}<0.001$. miR, microRNA; ALP, alkaline phosphatase; RUNX2, Runt-related transcription factor 2; NC, negative control.

of BMSCs and inhibited the expression of osteogenic marker genes.

GATA4 directly targets miR-144-3p. In our previous study (13), it was revealed that miR-144-3p was significantly downregulated during the osteogenic differentiation of BMSCs in rats and plays a negative regulatory role in this process. Huang et al demonstrated that miR-144-3p could affect the osteogenic differentiation process in rat BMSCs by regulating Smad4 (20), which demonstrated that Smad4 is a regulatory gene targeted by miR-144-3p; however, the regulatory factors upstream of miR-144-3p in the osteogenic differentiation process have not yet been studied, to the best of our knowledge. As mentioned above, GATA4 may be an upstream regulator of miR-144-3p. To further demonstrate the direct targeting relationship between GATA4 and miR-144-3p, an adenovirus infection experiment was conducted and dual-luciferase reporter gene detection was used.

BMSCs were infected by adenovirus carrying AdGATA4, and cell samples were collected after $48 \mathrm{~h}$ of infection for RT-qPCR detection. The mRNA levels of GATA4 and miR-144-3p were increased compared with those of the NC group (Fig. 3A and B). These differences were statistically significant, indicating that GATA4 had a positive regulatory effect on the expression of miR-144-3p. Next, whether GATA4 directly regulates miR-144 promoter activity was investigated. Two miR-144 promoter-luciferase plasmids containing 
A

miR-144-3p

Bank

AdGATA-4-NC
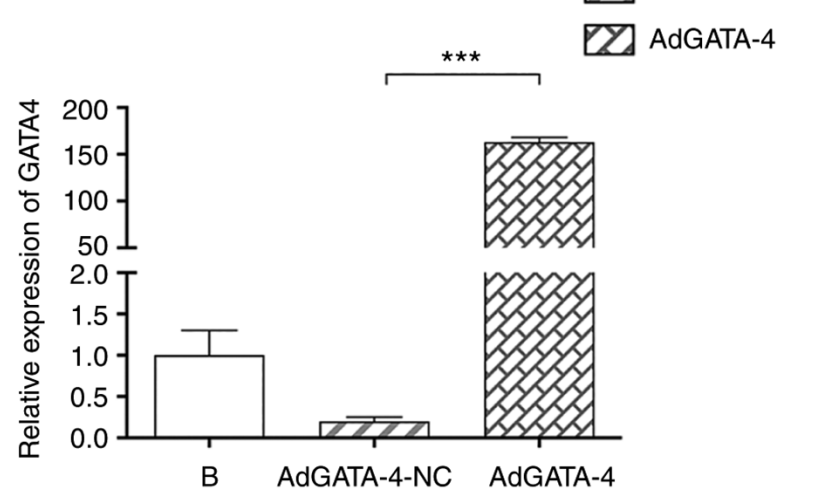

C miR-144
Promoter-luciferase constructs

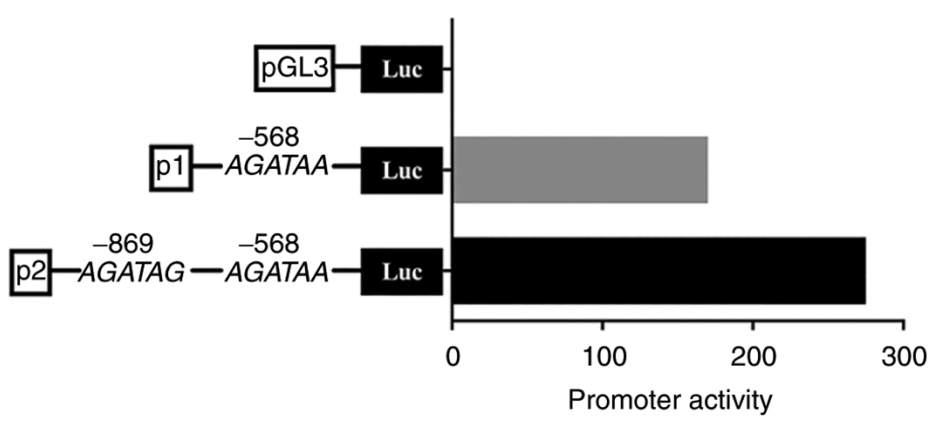

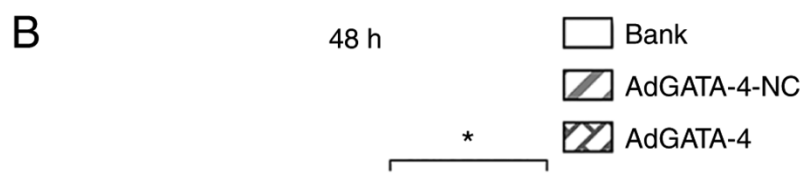
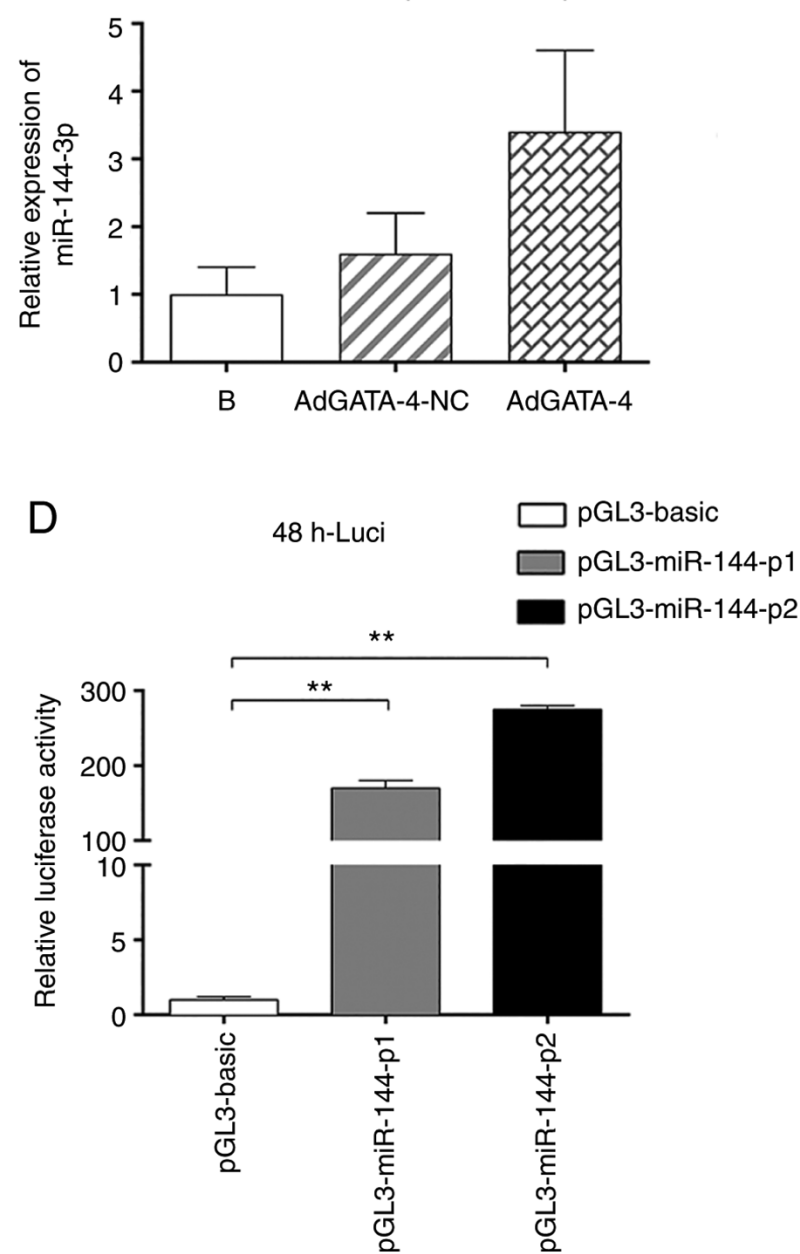

Figure 3. GATA4 has a positive regulatory effect on the expression of miR-144-3p and directly targets miR-144-3p. (A) RT-qPCR for detection of GATA binding protein 4 expression in AdGATA4-infected BMSCs. $\beta$-actin was used as the loading control. B group is a blank control (untransfected cells). (B) RT-qPCR for the detection of miR-144-3p expression in AdGATA4-infected BMSCs, with U6 used as the loading control. (C and D) Both -568 and -969 GATA sites were responsible for GATA4-induced activation of the miR-144 promoter in BMSCs. All data are the mean \pm SD from three independent experiments. ${ }^{*} \mathrm{P}<0.05$, ${ }^{* *} \mathrm{P}<0.01$ and ${ }^{* * *} \mathrm{P}<0.001$. miR, microRNA; RT-qPCR, reverse transcription-quantitative PCR; BMSCs, bone marrow mesenchymal stem cells; NC, negative control.

one (P1) or two (P2) putative GATA-binding sites at positions -568 and -969 of the miR-144 promoter region were generated (Fig. 3C). These luciferase reporter constructs and the pGL3-basic vector were cotransfected into $293 \mathrm{~T}$ cells with AdGATA4. After $48 \mathrm{~h}$ of transfection, the luciferase activity of plasmids P1 and P2 was significantly increased compared with that of the control transfection group. Moreover, plasmid P2, which contained two binding sites, exhibited stronger luciferase activity compared with plasmid $\mathrm{P} 1$, which contained one binding site (Fig. 3C and D). Therefore, the experimental results indicated that the GATA4 regulatory site played a positive role in regulating the regions of the miR-144 promoter at -568 and -969 .

\section{Discussion}

miRNAs are important posttranscriptional regulators confirmed by numerous studies to be involved in the regulation of bone metabolism $(21,22)$. In normal human serum, the type and amount of miRNAs are essentially stable. When tissues are destroyed, miRNAs in tissues will be released into the blood, causing an aberrant increase in the miRNA content in the serum $(23,24)$. In addition, when lesions occur in the body, such as those due to tumors and OP, the miRNA content in serum will also become aberrant $(25,26)$. Therefore, miRNAs can serve as biomarkers for the early diagnosis of OP.

Previous basic studies on OP have revealed that the selection of target miRNAs in BMSC osteogenic differentiation can be achieved by miRNA microarray detection and analysis of miRNA expression in BMSCs undergoing osteogenic differentiation (27) or by collecting blood samples from OP patients and healthy controls for the screening of target miRNAs by RT-qPCR (28). After a target gene was identified, cellular experiments in which the target gene was overexpressed or inhibited were conducted to further verify its role in BMSC 
osteogenic differentiation (29). In our previous study (13), miR-144-3p was selected as a target gene by assessing the first group of selected targets; determining the 10 most likely candidate target genes, again through the collection of blood samples from OP patients and healthy controls and RT-qPCR detection of mRNA levels of candidate target genes; and selecting the target genes with the greatest reliability, which revealed miR-144-3p as a target gene. In the present study, during osteogenic induction and differentiation, the expression of miR-144-3p was decreased, while the expression of osteogenic marker genes (ALP and Runx2) was increased. After further cell transfection, the expression of endogenous miR-144-3p was inhibited, osteogenic induction and differentiation continued, and the expression of osteogenic marker genes was increased. Overexpression of miR-144-3p inhibited the expression of osteogenic markers. These results indicated that miR-144-3p negatively regulated the osteogenic differentiation of BMSCs. Our previous study revealed that miR-144-3p expression was significantly reduced in the peripheral blood and bone tissue in patients with OP and that through targeting RANK, miR-144-3p regulated osteoclast formation, further affecting bone metabolism and the bone formation process, leading to the occurrence of OP (13). Research on miR-144-3p is limited to its targeting of RANK to regulate the osteoclast formation process, and the role of miR-144-3p in the differentiation of BMSCs has not yet been studied, to the best of our knowledge. Nevertheless, the regulatory factors upstream of miR-144-3p in the osteogenic differentiation process have not yet been studied, to the best of our knowledge.

In previous years, most studies on GATA4 have examined its role in regulating the differentiation of myocytes from BMSCs. GATA4 has been revealed to also play an important role in the regulation of bone metabolism, primarily by regulating the differentiation of BMSCs into osteoblasts $(30,31)$. Song et al demonstrated that GATA4 could negatively regulate osteogenic differentiation in BMSCs (17). Other previous studies have reported that GATA4 could not only inhibit the expression of RANKL in the process by which BMSCs differentiate into osteoblasts but also regulate the differentiation of osteoclasts (32-35). In addition, GATA4 has been revealed to participate in the regulation of tooth development by affecting the expression of osteogenesis regulatory factors and promoting the proliferation of tooth mesenchymal cells (36). Zhang et al reported that GATA4 could directly regulate the miR-144/451 gene cluster in the mechanism of myocardial ischemia and reperfusion and played a protective role in myocardial cells (37). To further study the roles of GATA4 and miR-144-3p in osteogenic differentiation, an adenovirus infection experiment was designed. Adenovirus carrying AdGATA4 was used to infect BMSCs, and RT-qPCR detection was conducted at $48 \mathrm{~h}$ after infection. The mRNA expression levels of AdGATA4 and miR-144-3p were increased compared with those in the NC group. Then, by dual-luciferase assay, it was revealed that the GATA4 regulatory site played a positive role in regulating the regions of the miR-144 promoter at -568 and -969 .

In conclusion, our results demonstrated that miR-144-3p played a negative regulatory role in the osteogenic differentiation of BMSCs and that GATA4 inhibited osteogenic differentiation by targeting the expression of miR-144-3p. However, the downstream mechanism of GATA4-mediated miR-144-3p regulation in osteogenic differentiation requires further investigation. OP is caused by the imbalance between bone formation and bone resorption (38). In the present study, only osteogenic differentiation was examined, and no relevant studies were conducted on osteoclast differentiation. Future studies will include in vivo experiments to investigate the effects of miR-144-3p on bone formation and bone resorption. Thus, further in vivo experiments are necessary to verify the clinical application of miR-144-3p in the treatment of OP.

\section{Acknowledgements}

Not applicable.

\section{Funding}

The present study was supported by grants from the National Natural Science Foundation of China (grant no. 82060271), the Guiyang Science and Technology Bureau of Guizhou Province (grant no. 2016-100146) and the Science Foundation of Guizhou Medical University (grant no. 19NSP046).

\section{Availability of data and materials}

All data generated and/or analyzed during the present study are included in the published article.

\section{Authors' contributions}

TQ and CW conceived and designed the experiments. TQ performed the experiments. TQ, HL, TL, LS and CC wrote the manuscript. HL, TL and CC made substantial contributions to conception and design, acquisition of data, and analysis and interpretation of data. LS was involved in drafting the manuscript and revising it critically for important intellectual content. LS designed the experiments. CW reviewed and revised the manuscript for important intellectual content. TQ and CW confirmed the authenticity of all the raw data. All authors have read and approved the final manuscript.

\section{Ethics approval and consent to participate}

Not applicable.

\section{Patient consent for publication}

Not applicable.

\section{Competing interests}

The authors declare that they have no competing interests.

\section{References}

1. Hendrickx G, Boudin E and Van Hul W: A look behind the scenes: The risk and pathogenesis of primary osteoporosis. Nat Rev Rheumatol 11: 462-474, 2015. 
2. Pouresmaeili F, Kamalidehghan B, Kamarehei M and Goh YM A comprehensive overview on osteoporosis and its risk factors Ther Clin Risk Manag 14: 2029-2049, 2018.

3. Paspaliaris V and Kolios G: Stem cells in osteoporosis: From biology to new therapeutic approaches. Stem Cells Int 2019: $1730978,2019$.

4. Liu J, Wu M, Feng G, Li R, Wang Y and Jiao J: Downregulation of LINC00707 promotes osteogenic differentiation of human bone marrow-derived mesenchymal stem cells by regulating DKK1 via targeting miR-103a-3p. Int J Mol Med 46: 1029-1038, 2020.

5. Shenoy A and Blelloch RH: Regulation of microRNA function in somatic stem cell proliferation and differentiation. Nat Rev Mol Cell Biol 15: 565-576, 2014.

6. van Wijnen AJ, van de Peppel J, van Leeuwen JP, Lian JB, Stein GS, Westendorf JJ, Oursler MJ, Im HJ, Taipaleenmäki H, Hesse E, et al: MicroRNA functions in osteogenesis and dysfunctions in osteoporosis. Curr Osteoporos Rep 11: 72-82, 2013

7. Nugent M: MicroRNA function and dysregulation in bone tumors: The evidence to date. Cancer Manag Res 6: 15-25, 2014

8. Clark EA, Kalomoiris S, Nolta JA and Fierro FA: Concise review: MicroRNA function in multipotent mesenchymal stromal cells. Stem Cells 32: 1074-1082, 2014.

9. Chen H, Ji X, She F, Gao Y and Tang P: miR-628-3p regulates osteoblast differentiation by targeting RUNX2: Possible role in atrophic non-union. Int J Mol Med 39: 279-286, 2017.

10. Cui X, Wang S, Cai H, Lin Y, Zheng X, Zhang B and Xia C: Overexpression of microRNA-634 suppresses survival and matrix synthesis of human osteoarthritis chondrocytes by targeting PIK3R1. Sci Rep 6: 23117, 2016

11. Chen J, Li K, Pang Q, Yang C, Zhang H, Wu F, Cao H, Liu H, Wan Y, Xia W, et al: Identification of suitable reference gene and biomarkers of serum miRNAs for osteoporosis. Sci Rep 6: 36347, 2016.

12. Panach L, Mifsut D, Tarín JJ, Cano A and García-Pérez M: Serum circulating microRNAs as biomarkers of osteoporotic fracture. Calcif Tissue Int 97: 495-505, 2015.

13. Wang C, He H, Wang L, Jiang Y and Xu Y: Reduced miR-144-3p expression in serum and bone mediates osteoporosis pathogenesis by targeting RANK. Biochem Cell Biol 96: 627-635, 2018.

14. Laforest B and Nemer M: GATA5 interacts with GATA4 and GATA6 in outflow tract development. Dev Biol 358: 368-378, 2011.

15. Zhou P, He A and Pu WT: Regulation of GATA4 transcriptional activity in cardiovascular development and disease. Curr Top Dev Biol 100: 143-169, 2012

16. Li Q, Shen P, Zeng S and Liu P: TIEG1 inhibits angiotensin II-induced cardiomyocyte hypertrophy by inhibiting transcription factor GATA4. J Cardiovasc Pharmacol 66: 196-203, 2015.

17. Song I, Kim K, Kim JH, Lee YK, Jung HJ, Byun HO, Yoon G and Kim N: GATA4 negatively regulates osteoblast differentiation by downregulation of Runx2. BMB Rep 47: 463-468, 2014.

18. Snykers $S$, Vanhaecke $T$ and Rogiers V: Isolation of rat bone marrow stem cells. Methods Mol Biol 320: 265-372, 2006.

19. Livak KJ and Schmittgen TD: Analysis of relative gene expression data using real-time quantitative PCR and the 2(-Delta Delta C(T)) method. Methods 25: 402-408, 2001.

20. Huang C, Geng J, Wei X, Zhang R and Jiang S: miR-144-3p regulates osteogenic differentiation and proliferation of murine mesenchymal stem cells by specifically targeting Smad4. FEBS Lett 590: 795-807, 2016.

21. Yuan Y,Zhang L, Tong X, Zhang M, Zhao Y, Guo J, Lei L, Chen X, Tickner J, Xu J, et al: Mechanical stress regulates bone metabolism through microRNAs. J Cell Physiology 232: 1239-1245, 2017.

22. Xie Y, Zhang L, Gao Y, Ge W and Tang P: The multiple roles of microrna-223 in regulating bone metabolism. Molecules 20 19433-19448, 2015.
23. Sugimachi K, Matsumura T, Hirata H, Uchi R, Ueda M, Ueo H, Shinden Y, Iguchi T, Eguchi $\mathrm{H}$, Shirabe $\mathrm{K}$, et al: Identification of a bona fide microRNA biomarker in serum exosomes that predicts hepatocellular carcinoma recurrence after liver transplantation. Br J Cancer 112: 532-538, 2015.

24. Witwer KW: Circulating microRNA biomarker studies: Pitfalls and potential solutions. Clin Chem 61: 56-63, 2015.

25. Wang X, Guo B, Li Q, Peng J, Yang Z, Wang A, Li D, Hou Z, Lv K, Kan G, et al: miR-214 targets ATF4 to inhibit bone formation. Nat Med 19: 93-100, 2013.

26. Matsumura T, Sugimachi K, Iinuma H, Takahashi Y, Kurashige J, Sawada G, Ueda M, Uchi R, Ueo H, Takano Y, et al: Exosomal microRNA in serum is a novel biomarker of recurrence in human colorectal cancer. Br J Cancer 113: 275-281, 2015.

27. Huang K, Fu J, Zhou W, Li W, Dong S, Yu S, Hu Z, Wang H and Xie Z: MicroRNA-125b regulates osteogenic differentiation of mesenchymal stem cells by targeting $\operatorname{Cbf} \beta$ in vitro. Biochimie 102: 47-55, 2014

28. Li Z, Zhang W and Huang Y: miRNA-133a is involved in the regulation of postmenopausal osteoporosis through promoting osteoclast differentiation. Acta Biochim Biophys Sin (Shanghai) 50: 273-280, 2018.

29. Chen T, Che X, Han P, Lu J, Wang C, Liang B, Hou Z, Wei X, Wei $\mathrm{L}$ and Li P: MicroRNA-1 promotes cartilage matrix synthesis and regulates chondrocyte differentiation via post-transcriptional suppression of Ihh expression. Mol Med Rep 22: 2404-2414, 2020.

30. Zhong L, Chiusa M, Cadar AG, Lin A, Samaras S, Davidson JM and Lim CC: Targeted inhibition of ANKRD1 disrupts sarcomeric ERK-GATA4 signal transduction and abrogates phenylephrine-induced cardiomyocyte hypertrophy. Cardiovasc Res 106: 261-271, 2015.

31. Song I, Jeong BC, Choi YJ, Chung YS and Kim N: GATA4 negatively regulates bone sialoprotein expression in osteoblasts. BMB Rep 49: 343-348, 2016.

32. Khalid AB, Slayden AV, Kumpati J, Perry CD, Berryhill SB, Crawford JA, Fatima I, Morselli M, Pellegrini M, Miranda-Carboni GA and Krum SA: GATA4 represses RANKL in osteoblasts via multiple long-range enhancers to regulate osteoclast differentiation. Bone 116: 78-86, 2018

33. Khalid AB, Slayden AV, Kumpati J, Perry CD, Osuna MAL, Arroyo SR, Miranda-Carboni GA and Krum SA: GATA4 directly regulates runx 2 expression and osteoblast differentiation. JBMR Plus 2: 81-91, 2018

34. Miranda-Carboni GA, Guemes M, Bailey S, Anaya E, Corselli M, Peault B and Krum SA: GATA4 regulates estrogen receptor-alpha-mediated osteoblast transcription. Mol Endocrino 25: 1126-1136, 2011.

35. Güemes M, Garcia AJ, Rigueur D, Runke S, Wang W, Zhao G, Mayorga VH, Atti E, Tetradis S, Péault B, et al: GATA4 is essential for bone mineralization via ER $\alpha$ and TGF $\beta / B M P$ pathways. J Bone Miner Res 29: 2676-2687, 2014.

36. Guo S, Zhang Y, Zhou T, Wang D, Weng Y, Wang L and Ma J: Role of GATA binding protein 4 (GATA4) in the regulation of tooth development via GNAI3. Sci Rep 7: 1534, 2017.

37. Zhang X, Wang X, Zhu H, Zhu C, Wang Y, Pu WT, Jegga AG and Fan GC: Synergistic effects of the GATA-4-mediated miR-144/451 cluster in protection against simulated ischemia/reperfusion-induced cardiomyocyte death. J Mol Cell Cardiol 49: 841-850, 2010.

38. Kureel J, Dixit M, Tyagi AM, Mansoori MN, Srivastava K, Raghuvanshi A, Maurya R, Trivedi R, Goel A and Singh D: miR-542-3p suppresses osteoblast cell proliferation and differentiation, targets BMP-7 signaling and inhibits bone formation. Cell Death Dis 5: e1050, 2014. 